\title{
COLLABORATION WITHIN THE TOOL-AND-DIE MANUFACTURING INDUSTRY THROUGH OPEN-SOURCE MODULAR ERP/CRM SYSTEMS
}

\author{
Ali Imtiaz \\ Research Institute for Operations Management (FIR) at Aachen University of Technology, \\ GERMANY \\ ali.imtiaz@fir.rwth-aachen.de \\ Jannicke Baalsrud Hauge \\ Bremen Institute of Industrial Engineering and Applied Work Science, GERMANY \\ baa@biba.uni-bremen.de \\ Si Chen \\ Open Source StrategiesINC \\ sichen@opensourcestrategies.com
}

\begin{abstract}
There is a definite trend for European SMEs to form "collaborative networks" based on core competencies to have a competitive advantage in the dynamic global market. This leads to the need of utilising electronic communication for which arrangements for a basic level of technological competence and IT infrastructure have to be established. Currently, the IT infrastructure of many SMEs is based on inappropriate tools, which cannot supports standardised communication within the network. The Tool-East initiative described in this paper is attempting to reduce the technological infrastructure hurdle by providing an open-source ERP/CRM platform to SME participants in the Tooland-Die making industry. This will be done in the hope that SMEs in this sector will standardise their business process annotation so that they' will be able to exchange business documents with each other electronically.
\end{abstract}

\section{INTRODUCTION}

Manufacturing has always represented a strong foundation for the European economy, reaching currently at about $28 \%$ of the European Gross Domestic Product (GDP), and presents an undoubted source of wealth. A strong manufacturing industry is certainly necessary to create a stable European employment market. From a European perspective, the integration of the production clusters is a very important prerequisite to secure the success of these manufacturing clusters in the global market.

One prominent sector within the manufacturing industry is Tool-and-Die manufacturing. Almost all Tool-and-Die workshops in Eastern Europe are small or medium sized enterprises (SMEs). Low margins and a decreasing market share force most of these SMEs to optimise productivity and structure business processes more efficiently. Furthermore, increasing competition requires professional customer relationship management and better cooperation with other companies in this sector. SMEs need to become more responsive to rapid fluctuation in supply and demand, to assure availability of different materials at the right time and at the right place, and to reduce inventory risks. To achieve these goals, Tool-and-Die workshops must bridge the gap between their order acceptance, inventory, and production processes. 
The success of collaboration is not only dependent on finding a partner with the right key competencies, but also about having appropriate ICT tools for seamless information processing and involving the right people to perform the daily collaboration work as well as on the ability of the participating organisations to act in a dynamical environment. Typical ICT tools needed for fast and seamless information processing within a networked environment are ERP and CRM tools. For intra-organisational collaboration, the newest SCM tools also provide some of the required functionalities. Until recently, such tools have been quite expensive to buy and their implementation required a high degree of IT competencies within the company. Even though large industrial enterprises play a dominating role in the European manufacturing industry, SMEs mostly acting as suppliers to larger ones still remain the backbone of the economy, especially in the Eastern European countries. However, if the SMEs intend to survive and to thrive, they need to stay competitive and to increase their productivity; therefore there is a need of a fast, reliable integrating support system at a feasible price fostering collaboration between SMEs. In order to keep the cost and the access on a low level, such a collaboration tool should be open-source based and only offer the needed functionalities. Therefore, the focus from uniform solution for all industries has to be shifted to Tool-and-Die making industry and their specific needs.

\section{MAIN RESEARCH CHALLENGES AND APPROACH}

Collaborations are complex to handle due to various reasons: different goals among collaborating partners and rapid process changes. In these dynamic and flexible networks, most partners have not collaborated before, which often results in the lack of trust. In addition, European collaboration networks also need to deal with the culture aspect. The above mentioned problems are relevant for almost all collaborations.

These problems can be classified into three categories:

- Interoperability, management and organisational models

- Sharing culture and technology transfer

- Cheap and effective software solutions based on open-source standards

For Tool-and-Die making workshops, the problems are mainly related to their production management and resource planning, customer relationship management, and especially e-collaboration. Collaboration processes represent a key factor in the competitiveness of Tool-and-Die making clusters. By improving these processes, clusters will be able to strengthen their position as suppliers. Such an improvement can be achieved through the implementation of a collaboration platform utilising emerging, innovative technologies at the lowest possible cost.

The EU-funded Tool-East project aims at developing an integrated solution for the overall supply chain in the Tool-and-Die making industry. In order to integrate a solution in the overall supply chain, the solution will connect all SMEs' existing individual back-end systems. The solution will be augmented with (adaptive) service modules, allowing fast and flexible, standardised interfacing. Different modules will be integrated to develop a synchronised open-source ERP application in close relationship with each SME in the consortium. 


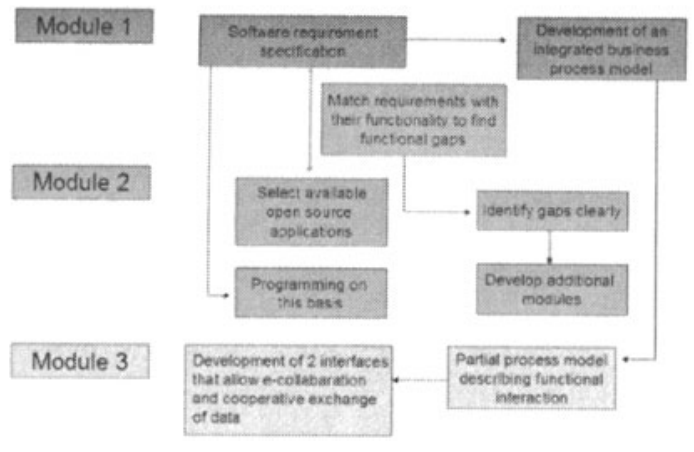

Module 1: Detinition of a ganenc business model tor tool and die making workshops

Modue 2: Open souce sotware architecture and application development

Module 3: Standand interfoces for open soure applications

Module 4: Findings of traing and education

Figure 1: Tool East Modules

\section{Module I: Definition of a generic business process model for Tool-and-Die making workshops}

Modelling the business process is an essential part of any information-based business networking development process. A survey was performed in the Tool-East project to model the business processes at the participating SMEs in order to develop software requirement specifications. The software requirement specification from the business model is formulated as the basis for software development, which specifies the required behaviour of a system.

The performed survey of suitable process models showed that the Aachner PPC [Luczak, 1999] model is the most suitable for analysing the end user business processes and to collect the user requirements. Based upon the individual business processes of the end-user's places, a semi-generic business process reference model was developed. The idea behind the model is the reduction of time needed for any new SME to analyse whether the Tool-East solution is suitable for them or not.

\section{Module II: Open source software architecture and application development}

The project will come up with an enriched open-source ERP/CRM system with additional functionalities. The first objective of the second module is to evaluate and select the best open-source solution available as the foundation for the intended specific development. The second objective is to develop additional modules to support processes described in the business process model. SMEs will be involved from a very early stage, where a first prototype will be available in order to receive a first feedback.

\section{Module III: Standard interfaces for open-source applications}

Competitive forces are driving technology efforts towards the information exchange within and across corporate boundaries via intelligent interfaces. Evolving new technologies provide an array of services to effectively design and develop integrated, collaborative solutions that connect different systems like ERP, CRM, or other internal systems between partners, vendors, and other service providers. For SMEs, not only the integration of large systems is problematic, but also the possibility to import existing data stored e.g. in Microsoft Excel files or other individually programmed databases, since this data must be entered manually. The 
technical possibilities and the latest developments will be examined and evaluated to provide additional functionality via interfaces.

\section{Module IV: Training and education}

The findings of the training and education module are critical to develop a methodology to carry out personnel training. The methodology should support the establishment of an appropriate training system between classroom, on-site, and elearning modules. Furthermore, the educational trainings have to meet the different stakeholder groups' individual requirements and training needs.

\section{RESULTS}

\subsection{Collaboration in SME Clusters}

Since SMEs have formed clusters to collaborate more efficiently, the understanding of such clusters and collaboration processes within the clusters is important to the Tool-East project. The approach followed within the Tool-East project analyses the individual SME requirements to support cluster evolution towards collaborative business. The analysis revealed that the development of a collaborative cluster followed an evolutionary path. The evolutionary paths of SMEs in these clusters and the cluster itself have three fundamental stages, represented in Figure 2:

- Empowerment - Cluster SMEs are very cost conscious and limit overhead costs, like administration and IT, to a bare minimum. In a first step, SMEs need to be empowered by low cost applications to support their key business processes. As a result of empowerment, reliable business data are available for internal use and for external integration.

- Integration - At this stage, the SME is able to open internal business processes to their main supply chain partners, either customers or suppliers. The perspective is still enterprise-centric, i.e. from inside the SME out. This is made possible through the adoption of data interchange standards and tools to support transactional processes for supply chain integration.

- Awareness - In the final stage, SMEs are aware that their business processes belong in the bigger picture of the overall cluster. The cluster behaves externally like a single enterprise, as SMEs are able to cooperate in knowledge-intensive and strategic processes like, e.g., sourcing, product development, marketing, and customer relationship management. 

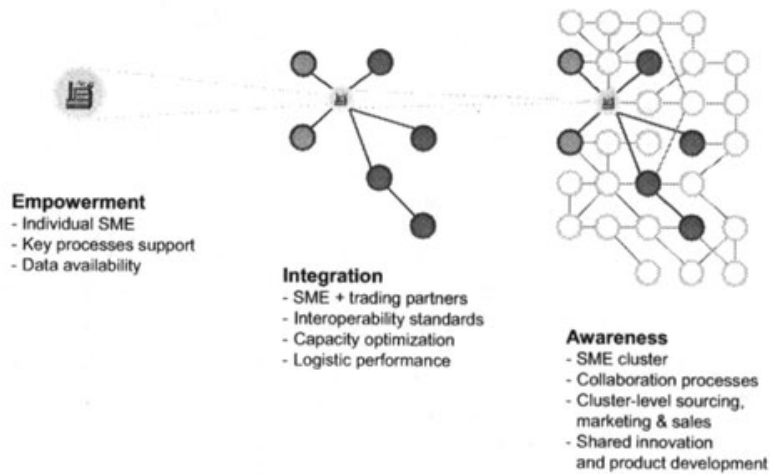

Figure 2: Stages in SME Cluster Evolution

By introducing the Tool-East concept, standardised processes will be introduced, which lead to better data exchange between business partners in electronic format. For the Tool-and-Die making clusters, the standardisation implies that they can take advantage of other e-business tools to support their particular business processes.

\subsection{Open Source Platform for Collaborative Software Development}

The term "open source" technically refers to software whose source code is made available under a license that permits its use, modification, and redistribution [OSI Definition]. In practice, such a licensing model has created the environment where software is developed collaboratively by a group of users and developers. Opensource software development projects share their source code through a source code repository management system such as Concurrent Versioning System (CVS) or Subversion (SVN), and the users and developers coordinate their efforts via an electronic mailing list, forum, or issue tracker.

Open source creates a collaborative environment in two significant ways. First, open-source development projects are themselves developed in a collaborative manner, with many users and developers contributing. Second, an open-source project can be extended to fit new requirements outside of its original goals. Thus, an open-source project can be thought of as a lump sum contribution by its original developers to a new software package.

In its ideal form, the collaboration between the original open-source project and the new package based upon or "derived" from it can be highly beneficial to both groups. The developers of the derived package can benefit from all the features offered by the original project plus continuous maintenance and enhancements for those core features over time. The original package could benefit from regular enhancements contributed back to it by the developers of the derived package. For this symbiotic relationship to occur, the following conditions must be met:

1. There must be compatible, mutually beneficial, but separate areas of focus and competency between the developers of the two packages.

2. The code of the derived package must be well separated from the original open source project, permitting regular updates.

3. The original project must provide stable releases for use by the developers of derived packages and regular updates for those developers to benefit from future enhancements. 
The developers of both packages must be committed to the symbiotic relationship.

\section{3 'Opentaps' ERP System as a Collaborative Platform}

The Tool-East project is geared towards the extension of restrictive and limited ERP systems to incorporate standardised collaboration processes between SMEs. A Uniform application platform will include features focusing on interoperability and communication required for collaboration. The extension focuses on two points: 1) the development of a strict intercommunication standard for different companies with different ERP systems to communicate, and 2) the flexibility through opensource application allowing the users to further extend the features without altering the company's base application. To extend the features of an ERP system, a modular approach must be taken. Modular ERP systems have open system architectures, which means any module can be interfaced or detached whenever required without affecting the other modules. Furthermore, such modular systems support third party add-ons and multiple hardware platforms for the companies having a heterogeneous collection of systems and support.

As a preferred solution, Opentaps (www.opentaps.org) was selected to be customised according to the requirements of Tool-and-Die makers. Opentaps is an open-source ERP and CRM application suite based upon the Apache OFBIZ framework and is a Java-based application. The software leverages the technically robust Apache OFBIZ framework, which in turn incorporates such well-known open-source projects as Tomcat, Lucene, Derby, and Xerces, to build a complete ERP and CRM application. Like many open-source projects, Opentaps is developed within an open-source community, with the project manager Open Source Strategies, Inc. serving as the hub of the network.

By incorporating Opentaps into an industry-specific ERP/CRM, such as one for European Tool-and-Die making SMEs, the developers of the industry-specific package gain the benefits of collaborating with the users and developers of the Opentaps open-source community. Opentaps offers a technical infrastructure that addresses requirements common to all business applications and a core feature set that is based on international standards. Furthermore, an ongoing process of collaboration with Opentaps should bring continued maintenance and enhancements of both the infrastructure and the applications.

In practice, Opentaps has a modular components-based software architecture that facilitates collaborative development. Opentaps is divided into many smaller applications, each with its own data model, business logic, workflows, and user interface. Because each application component is kept completely separated, Opentaps allows add-on features to be implemented in a separate component with minimal overlap. New tables, business logic, screens, and security permissions can be created separately, and existing workflows can be altered with minimal change to the core code base. A developer of an industry-specific ERP/CRM system using Opentaps could take an existing release and add a new module according to the industry-specific requirements. Such an approach creates a de facto long-term collaboration between the developers of the industry-specific ERP/CRM system and the Opentaps open-source community. 


\section{EXPECTED BENEFITS FOR TOOL-AND-DIE MAKING CLUSTERS}

The results of the Tool-East project are expected to generate benefits for SMEs in the Tool-and-Die making industry by improving the cooperation in industrial clusters through an information platform. Serving as a collaborative platform, the developed ERP/CRM system will integrate the information flows between SMEs within an industrial cluster. This will allow the industrial cluster to reach the awareness stage in the SME cluster evolution. The ability for the cluster to behave externally like a single enterprise will generate a competitive advantage for the involved SMEs over independent SMEs. The expected competitive advantage should be observable through:

1. Quicker response to market demands due to a shortened time-to-market through improved communication and collaboration

2. Improved product quality through automatic information sharing e.g. CAD drawings and utilisation of the core competence of the individual SMEs

3. Cost reduction through optimised collaborative business processes and competence sharing.

\section{CONCLUSIONS AND SUMMARY}

SMEs in Europe have to develop innovative business strategies, for example, the participation in business networks and clusters, to secure long-term success. ERP/CRM applications and their integration with existing applications are necessary to ensure successful electronic collaboration in the business network. Applications must be extended with modules specifically created for the particular processes carried out in the cluster to enable the e-collaboration of SMEs in industrial clusters. Cost-efficient ERP applications based on existing open-source ERP applications represent a promising approach to foster collaboration in industrial clusters of SMEs. The temporal cluster Tool-East project with Tool-and-Die makers, IT companies and research institutes is exploring, selecting and adapting an opensource ERP application for exactly this purpose. The new adapted and modified ERP application will support the efficient coordination of intra-enterprise order processing and strengthens the competitiveness of Eastern European SMEs.

An enormous potential can be generated for SMEs through the development of an ERP application based on the open-source initiative. The expected benefits include cost advantages through an optimisation of collaboration, fast response to challenges, and a gradual reorganisation and simplification of complex information systems in the network. The benefits from a customised ERP/CRM solution thus improves three major factors for competition: 1) reduction of the time-to-market through improved communication, 2) higher quality through automatic information sharing e.g. CAD drawings, and 3) reduction of costs through optimised business processes.

The systematic and analytic approach used by the Tool-East project is not limited to the Tool-and-Die making industry. Through the generalisation of the approach, the impact can be replicated to different sectors for SMEs. The only prerequisite for applying the Tool-East approach for other sectors is the business process model. Based on the model, the selection and customisation of an ERP 
application for SMEs is possible. Such an approach is expected to bring similar advantages for SMEs in other industrial sectors.

For the long term success of European SMEs (like the Tool-and-Die making industry) it is essential to focus on knowledge intensive, innovative business strategies to produce goods and services that can compete on dynamic and global markets. Our research highlights the value of a strategy using IT technologies to increase participation in dynamic business networks as a way to achieve this.

\section{ACKNOWLEDGEMENTS}

This work has been partly funded by the European Commission through IST Project Tool-East: Open Source Enterprise Resource Planning and Order Management System for Eastern European Tool-and-Die Making Workshops (No. IST-FP6027802). The authors wish to acknowledge the Commission for their support. We also wish to acknowledge our gratitude and appreciation to all the Tool-East project partners for their contribution during the development of various ideas and concepts presented in this paper.

\section{REFERENCES}

1. [OSI Definition]: The Open Source Initiative: http://www.opensource.org/docs/definition.php

2. [Sherman, 1996]: Sherman, Heidemarie(1996): "Globalisierung: Transnationale Unternehmen auf dem Vormarsch", ifo Schnelldienst, Nr. 23, 1996

3. [Meyer, 2004]: Meyer, M et al(2004): Plug and do Business- ERP of the next generation for efficient order processing in dynamic business networks in: International Journal of Internet and Enterprise Management, p.153 Vol.2, 2004

4. [Luczak, 1999]Holger Luczak, Walter Eversheim: Produktionsplanung und -steuerung: Grundlagen, Gestaltung und Konzepte. 2. Auflage.: Springer-Verlag (1999), ISBN 3-540-65559-X

5. [Oliveira, 2000]: Oliveira et al. (2000) 'SMARTISAN-moving e-commerce to extended product', Helsinki Conference Proceedings.

6. [Scheer, 2002]: Scheer, A.-W., Grieble, O., Hans, S., Zang, S.(2002): Geschäftsprozessmanagement - The 2nd wave. In: Information Management \& Consulting, 17, 2002 Sonderausgabe, pp. 9-14.

7. [Sherman, 1996]: Sherman, Heidemarie(1996): “Globalisierung: Transnationale Unternehmen auf dem Vormarsch", ifo Schnelldienst, Nr. 23, 1996

8. [SCC2006)Supply Chain Council: SCOR 7.0 overview booklet. More Information will be found on http://www.supply-chain.org/page.ww?section=SCOR+Model\&name=SCOR+Model 